\title{
Evaluation of a Cable-Driven Parallel Robot: Accuracy, Repeatability and Long-Term Running
}

\author{
Marceau Métillon ${ }^{1,2}$, Nicolò Pedemonte ${ }^{3}$, and Stéphane Caro ${ }^{1,2}$ \\ 1 Laboratoire des Sciences du Numérique de Nantes (LS2N), UMR CNRS 6004, \\ 1, rue de la Noë, 44321 Nantes, France \\ $\{$ marceau.metillon, stephane.caro\}@ls2n.fr \\ 2 Centre National de la Recherche Scientifique (CNRS), \\ 1, rue de la Noë, 44321 Nantes, France \\ 3 IRT Jules Verne, Chemin du Chaffault, 44340, Bouguenais, France \\ nicolo.pedemonte@irt-jules-verne.fr
}

\begin{abstract}
The performance evaluation of Cable-Driven Parallel Robots in terms of accuracy, repeatability and long term capacities is a key for their industrial deployment. This paper presents and analyses corresponding experimental results for the PACE prototype, a fully-constrained, redundantly restrained, eight cables and six degrees of freedom cabledriven parallel robot. First, a long-term running test is carried out to evaluate the deviation of the moving-platform pose along time of use. A second experiment consists into a full design of experiments to evaluate the effect of some factors specific to this type of robot on its absolute accuracy and repeatability. Experimental results are presented and discussed.
\end{abstract}

Keywords: parallel robot · cable · accuracy · repeatability · long-term running

\section{Introduction}

Several industrial sectors, e.g. the naval and renewable energy industries, are facing with the necessity to manufacture and manipulate large and complex shape products. Cable-Driven Parallel Robots (CDPRs) are suitable for this type of application thanks to their high payload-to-weight ratio, large workspace and reconfiguration capabilities.

CDPRs are a particular class of parallel robots whose moving-platform (MP) is connected to the robot fixed base frame by a number of cables. The cables are coiled on motorized winches which control the cable lengths. The cables are routed from the winches to the MP through pulleys. The connection points between the cables and the base frame are referred to as exit points and are located on the exit pulleys and the connection points between the cables and the MP are referred to as anchor points. 
The knowledge of the accuracy and repeatability of CDPRs is a key feature to guarantee their correct fitting to the industrial task they are assigned to. Various approaches are proposed to improve the accuracy of CDPRs such as the use of additional sensors and data fusion. In [5,6], cable angle measurement sensors are used in a sensor fusion to improve the MP pose accuracy. In [8], a fusion of data from a camera and three laser distance sensors is used as a feedback in a closed-loop Cartesian pose controller. In [10], various combinations of sensor measurements such as IMU and cable angle are studied while in [12], cable tension sensors are used to simplify and solve the analytical Direct Kinematic (DK) problem of CDPRs. In [13], the estimation of the MP mass and payload using cable tension is studied. In [18] various algorithms and filters are considered in IMU-sensors fusion for solving the DK problem of a Gough-Stewart platform. In [11], three lidars are embedded on the MP during long-term running of a CDPR and are used to correct the Cartesian MP pose.

The use of elastic cable models also increases the robot accuracy. In [17], the use of a simple elastic cable model is implemented and experimentations exhibit an improved position accuracy. In [2], the use of an elasto-dynamic model of a non-redundant CDPR is studied in term of position accuracy improvement. In [14], the stiffness of the robot is studied and a Stiffness Oriented Tension Distribution Algorithm reduces the deviation of the MP under external wrenches. Exteroceptive vision-based control algorithms also contribute to the improvement of CDPR accuracy. In [4], a 3D pose kinematic visual servoing and a vision-based computed torque control were implemented for the control of a CDPR. In [3], a vision is used to track the robot position using tracked visual features placed on the MP and an external fixed camera. In [20], a $2 \frac{1}{2} \mathrm{D}$ visual servoing is studied and implemented experimentally on a CDPR to increase its robustness to manufacturing and calibration errors as well as to improve its accuracy.

Once designed and manufactured, an experimental evaluation allows one to determine the prototype accuracy and repeatability performances. In [15] the long-term running of a CDPR printer was analyzed. After a week of continuous running the joint lag was measured exhibiting the cable creep. In [7] the position accuracy of a three-dof CDPR performing dynamic trajectories was evaluated using an external vision tracking system. In [16], the vertical position error of a cable-based robotic crane were determined, a distribution of position errors along horizontal axes was also described and analysed. Others techniques can be used to increase CDPR accuracy. In [1], a Neural Network uses IMU sensor data to control the robot and images taken by an embedded camera are used to close the control loop. In [9], the cable elasticity is taken into account in the fusion of data from embedded IMU and encoder information.

In this paper, the accuracy and repeatability of a CDPR prototype, named PACE and located at IRT Jules Verne, are analyzed experimentally through long-term running. Furthermore a full factorial design of experiments is run to evaluate the effect of factors specific to CDPRs on the MP pose accuracy and repeatability. The paper is organized as follows: Section 2 presents the experi- 
mental setup considered in this work. Section 3 describes the method and shows the results obtained with the long-term experiment. Section 4 presents the fullfactorial design of experiments and the obtained results. Conclusions and future work are drawn in Sec 5.

\section{Experimental setup}

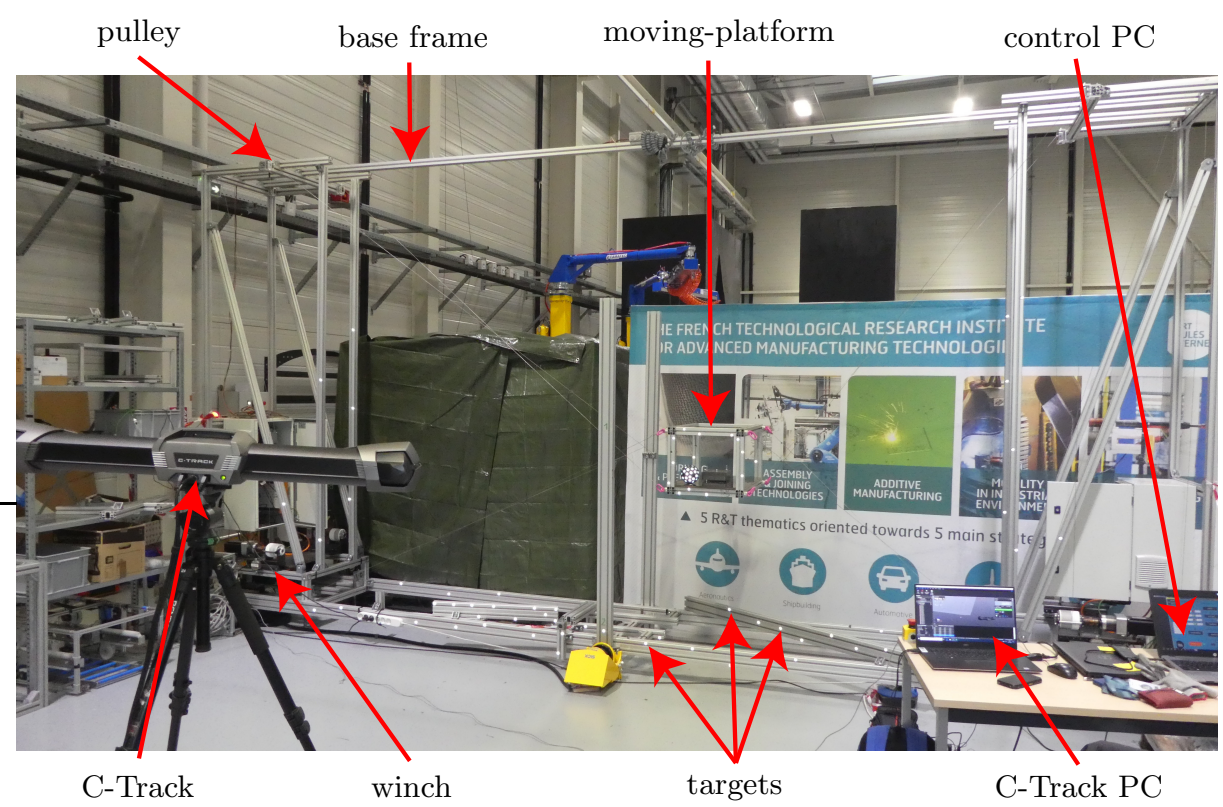

Fig. 1: PACE prototype and measurement device

\subsection{Robot prototype}

Figure 1 shows the fully-constrained CDPR under study named PACE and located at IRT Jules Verne, France. It is composed of eight cables and the size of its frame is: $L=4.6 \mathrm{~m} \times l=0.9 \mathrm{~m} \times H=2.8 \mathrm{~m}$. The cables are synthetic Dyneema ${ }^{\circledR}$ which reference is DYNE02SE99 ${ }^{4}$ with a nominal diameter of $2 \mathrm{~mm}$. Eight motorized winches are mounted on the frame. The coiling drum of the winches have grooves so that a single layer is coiled around the nominal diameter of the drums equal to $90 \mathrm{~mm}$. The cable length measurement estimation is obtained using the motor encoder with a resolution of $0.001 \mathrm{deg}$. Eight force sensors are located between the base frame and the respective eight winches

\footnotetext{
${ }^{4}$ Acquired from Corderie Lancelin
} 
in order to measure the cable tensions. Cables are routed through pulleys and connected to the vertex of a parallelepiped aluminium profile MP. Two control cabinets are mounted on each side of the frame and connect all the motors and needed equipment such as motors drivers, sensors, power supply and communication devices. A control PC is located in the switching cabinets. Using a remote connexion, it is possible to control the robot and download recorded data from the robot controller PC.

\subsection{Exteroceptive measurement}

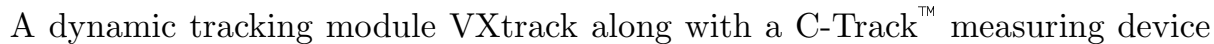
from Creaform are used to measure the MP pose. The device is composed of a set of two cameras mounted on a support and connected to a controller communicating with a computer for data acquisition. Self-adhesive circular dots, of diameter equal to $20 \mathrm{~mm}$, called targets are placed on the rigid main frame of the robot and identified as reference targets. The latter are used to define the global frame. Targets are also placed on the MP and on a faceted 3D-printed sphere fixed to the MP. They are identified as tracked targets and are used to measure the MP pose in the global frame of the robot at a rate of $30 \mathrm{~Hz}$. The measurement repeatability and accuracy are equal to $0.02 \mathrm{~mm}$ and $0.1 \mathrm{~mm}$, respectively within a $16 \mathrm{~m}^{3}$ measurement volume.

\section{Long-term running experiments}

The long-term running evaluation of the robot refers to the evaluation of the deviation of the MP pose during long-term on-duty and off-duty periods of the robot. The MP deviation amounts to the MP absolute accuracy, namely the difference between the desired MP pose and the measured MP pose.

\subsection{Method}

In this experiment, the robot is controlled using a classical closed-loop positionbased joint-space control. The desired trajectory motion described in operational space is converted into a set of joint coordinates using the Inverse Geometric Model (IGM) of the robot. A Feed-Forward model determines the needed joint torques to fulfil the static and dynamic equilibrium of the MP along the trajectory. In addition to this open-loop control architecture a feedback controller uses joint position information to compute a correcting torque to ensure the trajectory tracking without error between the desired trajectory and the trajectory followed by the robot in the joint space. The difference between joint position measurement and desired joint position (the joint error) is used in a proportionnal-integral-derivative (PID) feedback controller.

A path ${ }^{5}$ alternating translation and orientation movements of the MP was specifically defined for the long-term running experiments and is shown in Fig-

\footnotetext{
${ }^{5}$ Here is a link to the trajectory followed by the CDPR MP during long-term experiments: https://metillon.net/CableCon2021
} 


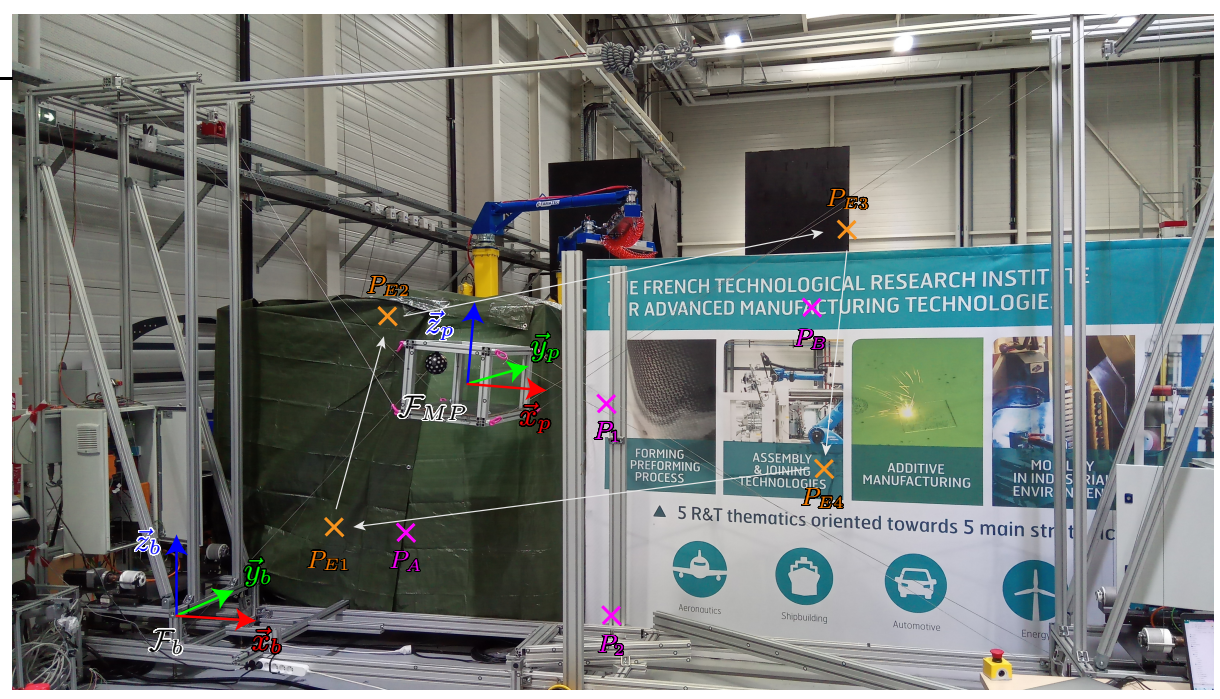

Fig. 2: Test path, targets and MP home poses

ure 2. The path is composed of four segments passing through the waypoints $P_{E 1}$, $P_{E 2}, P_{E 3}$ and $P_{E 4}$. The Cartesian coordinates of these four points expressed in the global frame $\mathcal{F}_{b}$ are the following :

$$
\begin{aligned}
& \mathbf{p}_{E 1}=[1.000,0.346,0.400]^{T} m \\
& \mathbf{p}_{E 2}=[1.500,0.346,1.200]^{T} m \\
& \mathbf{p}_{E 3}=[3.200,0.346,1.400]^{T} m \\
& \mathbf{p}_{E 4}=[2.800,0.346,0.800]^{T} m
\end{aligned}
$$

The orientation of the MP remains constant once the MP translates. The MP performs pure rotations at points $P_{B}$ and $P_{D}$. Accordingly, the test-trajectory is as follows:

1. Starting at point $P_{E 1}$, translation to point $P_{E 2}$.

2. Rotation of the MP of +5 deg then $-3 \operatorname{deg}$ around axis $\vec{x}_{b},+5 \operatorname{deg}$ then -5 deg around axis $\vec{y}_{b}$ and +5 deg then -5 deg around axis $\vec{z}_{b}$ successively while staying at position $P_{E 2}$

3. Translation from $P_{E 2}$ to $P_{E 4}$ via $P_{E 3}$

4. Rotation of the MP of +5 deg then -3 deg around axis $\vec{x}_{b},+5$ deg then -5 deg around axis $\vec{y}_{b}$ and +5 deg then -5 deg around axis $\vec{z}_{b}$ successively while staying at position $P_{E 4}$

5. Translation from $P_{E 4}$ to $P_{E 1}$

6. Pause of 10 seconds at point $P_{E 1}$

This trajectory is running on a loop all along the experiment. The MP velocity follows a trapezoidal profile along each segment and the maximum velocity is $1 \mathrm{~m} / \mathrm{s}$. One loop takes $40 \mathrm{~s}$ to complete. 
At the beginning of the experiment, the robot is calibrated at its home pose once and is then controlled to reach the initial point of the specific trajectory. At this point a first measurement of the MP pose is performed and is used as a reference for further measurements. From this point, the robot is expected to work three 8-hour work sessions on three different days without re-calibration. During a session, the robot repeats the trajectory on a loop during the whole session. At each iteration of the loop, the robot does stop during ten seconds at the initial point. This allows the exteroceptive measurement of the MP pose after settling of dynamic vibrations.

During sessions, three measurements are performed namely at the beginning, at the middle and at the end of the session. At the end of the session, once the measurement is made, the trajectory is stopped and the MP remains at the initial pose of the trajectory. The robot is then safely shut down using the brakes and standard procedure awaiting next session. At the beginning of the next session, the robot is powered on. The MP pose is measured in order to determine the static deviation occurring during the off duty period between the two sessions. Then the same procedure is repeated as per the first session for the second and third sessions.

\subsection{Long-term running experimental results}

At the end of the experiment, nine pose measurements of the MP were recorded corresponding to the beginning, the middle and the end of all three sessions as shown in Figure 3. The boxes represent the working session (namely on-duty) and the part of the graph outside the boxes represents the periods when the MP was held in position at initial point and not moving (namely off-duty).

By analyzing the different MP pose measurements it is possible to distinguish two different deviations. One is referred to as dynamic deviation which is accumulated during running hours of the robot, the other is referred to as static deviation and consists into the deviation added up during off-duty periods. Figure 3 shows the static and dynamic position and orientation deviations of the MP along time on the overall experiment. Figure 4 describes the part of the deviation obtained during on-duty and off-duty periods. The results exhibit a creep phenomenon of the cable during off-duty periods. As far as we understand, this is mainly due the MP mass exerting cable tensions and thus a permanent elongation of the cables. The opposite sign in the deviations comes from the routine used to stop the robot at the end of each running period. The trajectory is stopped when the robot comes back to the initial pose. The measurement at the end of the day is performed then the brakes are activated and the drivers stopped. Once the off duty period is over, in the beginning of the next running period, the robot is powered on by activating drivers and then releasing the brakes while servoing the motors on the current position. 


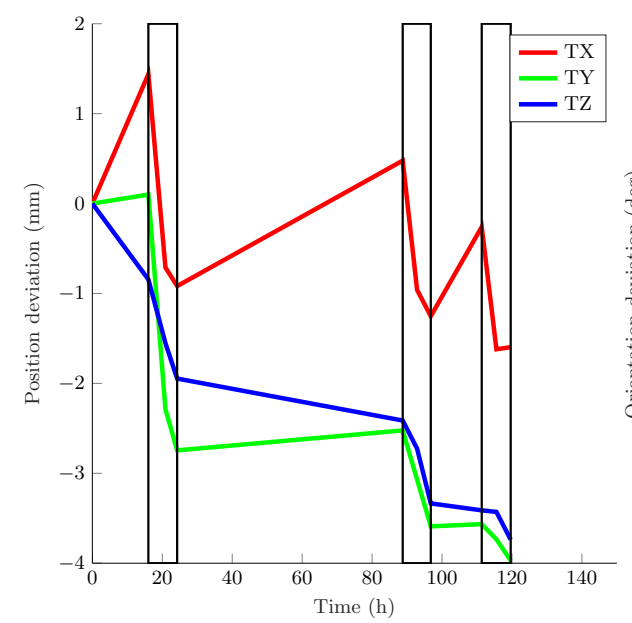

(a) Translational displacement

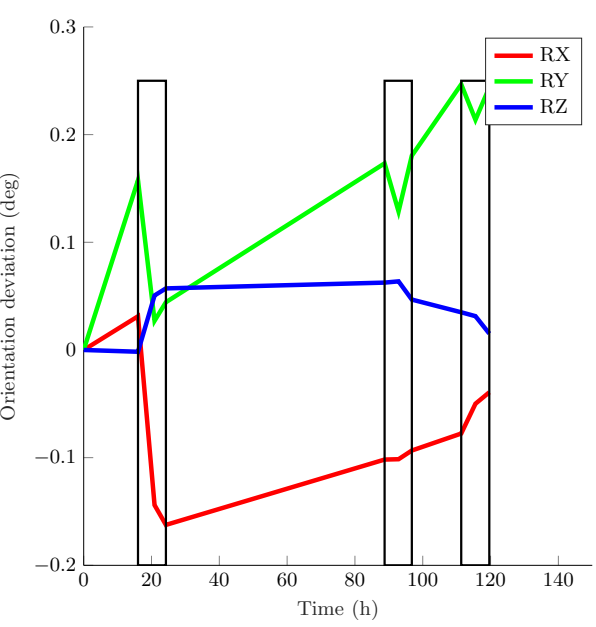

(b) Rotational displacement

Fig. 3: MP pose deviation along the three eight-hour sessions and during rest periods $($ Total time $=120 \mathrm{~h}=5$ days $)$
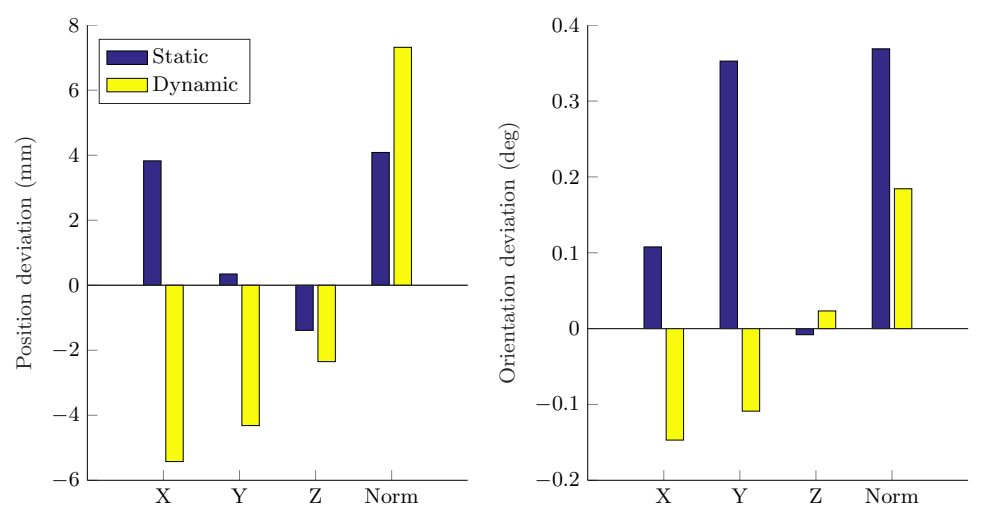

Fig. 4: Static and dynamic MP position and orientation deviations 


\section{Accuracy and repeatability}

This section aims to evaluate the absolute accuracy and repeatability of the prototype. The absolute accuracy is the capability of the robot to position its MP at a desired pose. The repeatability is the ability of the robot to repeatedly return to the same location under the same program with the same conditions [19]. Moreover, this section aims to determine the influence of factors specific to CDPR on the accuracy and repeatability of the MP pose.

\subsection{Method}

In order to identify the specific influence of each factor, a full factorial design of experiments is considered over the two-levels of each factor. The considered factors are the following : cable tension, home MP pose, elasticity and payload. Each factor has two-levels as described in Table 1.

Table 1: Full factorial design factors

\begin{tabular}{ccc}
\hline \hline factors & low level $(-1)$ & high level (1) \\
\hline cable tension & $50 N$ & $28 N$ \\
home MP pose & $\mathbf{p}_{1}=\left[\begin{array}{c}2.177,0.349,1.083]^{T} \mathrm{~m} \\
\text { elasticity }\end{array}\right.$ & $\mathbf{p}_{2}=[2.127,0.423,0.017]^{T} \mathrm{~m}$ \\
payload & without & with \\
without $(0 \mathrm{~kg})$ & with $(8 \mathrm{~kg})$ \\
\hline \hline
\end{tabular}

Considering the four factors, the full factorial design of experiment has $2^{4}=$ 16 different experiments. In order to take into account the repeatability, all experiments are repeated five times. Therefore 80 experiments are carried out in a randomised order.

\subsection{Factors}

The home MP pose factor defines the pose where the MP is calibrated. Two different calibration poses are considered, the first pose, named $P_{1}$, is located around the centre of the robot workspace while the second, named $P_{2}$ is located at the bottom of the robot workspace. The cable tension factor corresponds to the initial cable tension set. The initial cable tension is the parameter defining the amount of tension given to the cable during the calibration phase. During the calibration procedure, the MP is located at its home pose then the initial cable tension is gradually applied simultaneously to all cables. This ensures a certain pre-tension in the cable. Two sets are defined, each yielding the same initial cable tension for all cables. The elasticity factor defines whether the cable elasticity compensation algorithm of the prototype is used for the experiment or not. The cable elasticity compensation algorithm aims to compensate the cable elongation under the cable tension due to its elasticity. Using the cable 
tension and length information, a linear elastic cable model is used to determine the cable elongation, which is then used in the robot geometric model so that the desired cable length accounts for the elongation. The elasticity of the cable was identified experimentally such that $E S=121775.96 \mathrm{~N}$. The payload factor corresponds to the presence or the absence of the payload on the MP.

\subsection{Experimental procedure}

Two points of interest, known as target points, called $P_{A}$ and $P_{B}$ whose coordinates expressed in the base frame $\mathcal{F}_{b}$ are $\mathbf{p}_{A}=[1.000,0.346,0.400]^{T} \mathrm{~m}$ and $\mathbf{p}_{B}=[3.200,0.346,1.400]^{T} \mathrm{~m}$ are defined in the robot workspace. The pose of the MP is to be evaluated at these two points and compared to the desired pose. The accuracy can then be expressed as the mean of the error between the desired and measured poses over the five repetitions of a given experiment. The repeatability is the standard deviation of the error over the repetitions. For each experiment, the procedure is the following:

1. The platform is placed on the calibration point corresponding to the home MP pose factor $\left(P_{1}\right.$ or $\left.P_{2}\right)$.

2. The load corresponding to the payload factor is mounted on the platform (with or without).

3. The elasticity algorithm is set corresponding to the elasticity factor (with or without).

4. The initial cable tension set corresponding to the cable tension factor (high or low) is gradually applied to the cables in increments of $25 \%$ of the final value.

5. The robot reaches the target $P_{A}$ and waits.

6. The MP pose is measured using the $\mathrm{C}$-Track $^{\mathrm{TM}}$ measurement system.

7. The robot reaches the target $P_{B}$ and waits.

8. The MP pose is measured using the $\mathrm{C}-T r a c k^{\mathrm{TM}}$ measurement system.

9. The robot measurements are recorded.

10. The MP returns to the calibration point.

In this experiment, the control architecture is close to the one used in Sec. 3. The only difference lies in the cable elasticity compensation algorithm used, which is a function of the state of the cable elasticity factor.

\subsection{Results}

As per the exteroceptive measurements, the pose error is obtained as the difference between the desired pose and the measured pose of the MP. For a given experiment, the error is evaluated for each of the five repetitions at the two target positions $P_{A}$ and $P_{B}$. Figure 5 represents the MP pose error in terms of position and orientation error at $P_{A}$ for the first experiment configuration. Similarly, the same information is obtained concerning the pose $P_{B}$ but is not depicted in this paper for the sake of shortness. In this case the configuration is: home MP pose 
factor is set to $P_{1}$, the cable tension factor is set to high, the cable tension factor is set to high and the elasticity factor is set to without. Position and orientation error at points $P_{A}$ and $P_{B}$ constitute the responses of the full-factorial design and are collected for the 80 experiments constituting the full-factorial design.
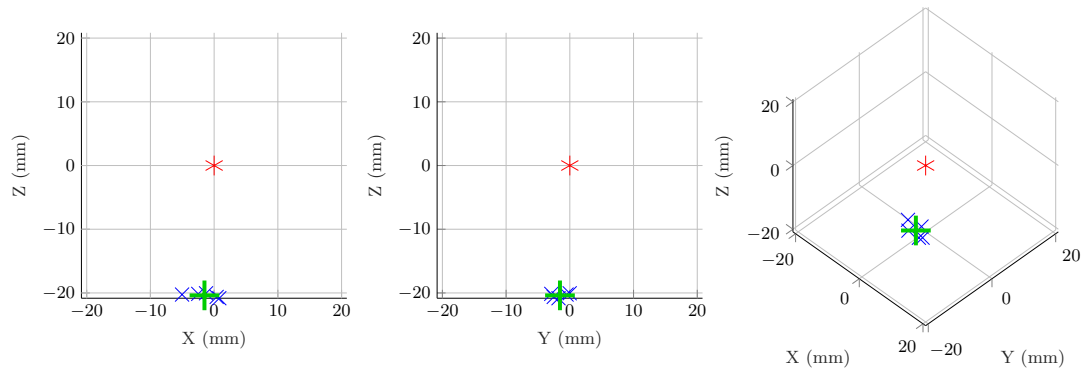

(a) Measured positions at point A
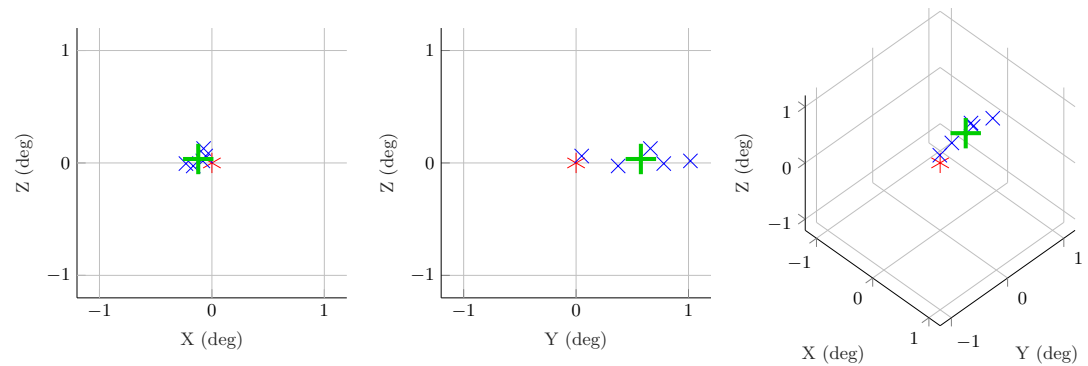

(b) Measured orientations at point $\mathrm{A}$

Fig. 5: MP accuracy and repeatability at Experiment 1: the desired target in red; the measured poses in blue; the mean error in green

Figure 6 shows the results in terms of accuracy and repeatability of the MP position at both points $P_{A}$ and $P_{B}$ for each of the sixteen experiments of the full factorial design. Likewise, the information on accuracy and repeatability in terms of MP orientation is obtained but not given in the paper for conciseness. For a good understanding of the effect of each factor and as well as the effect of interactions between factors on the robot accuracy and repeatability a factorial regression analysis was performed using the Minitab ${ }^{\circledR}$ software. Figure 7 shows the effects of each factor on the MP accuracy and repeatability. The factors influencing the accuracy are evaluated using a factorial regression on the mean of the error of the repetitions of the experiments while the factors influencing the repeatability are evaluated using a variability analysis of the error over the repetitions. It should be noted that the most influential factors on the robot accuracy are the home MP pose factor and the payload ones as well as their interaction with other factors. 


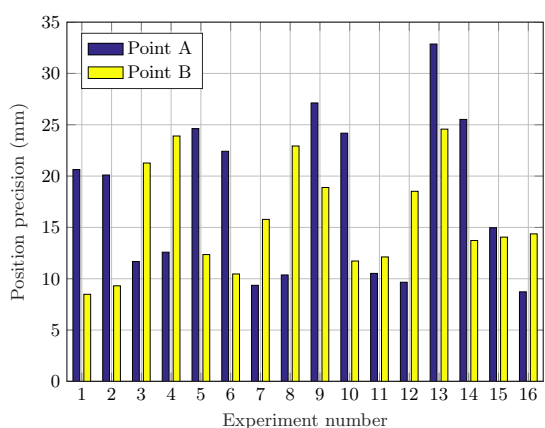

(a) Accuracy

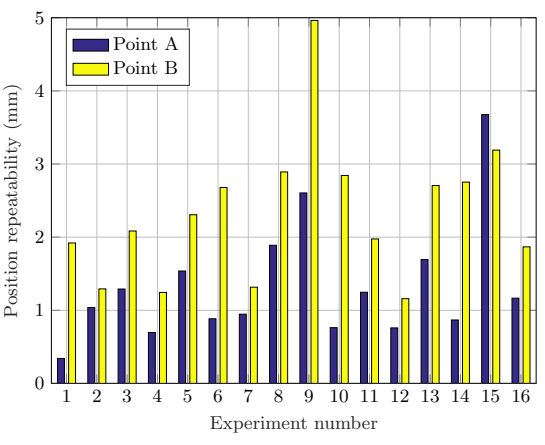

(b) Repeatability

Fig. 6: Position accuracy and repeatability for each experiment

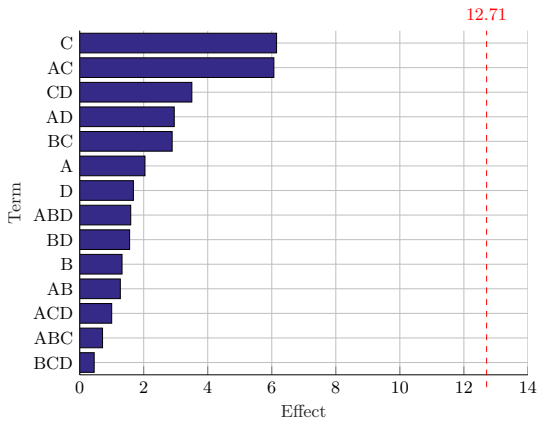

(a) Position accuracy

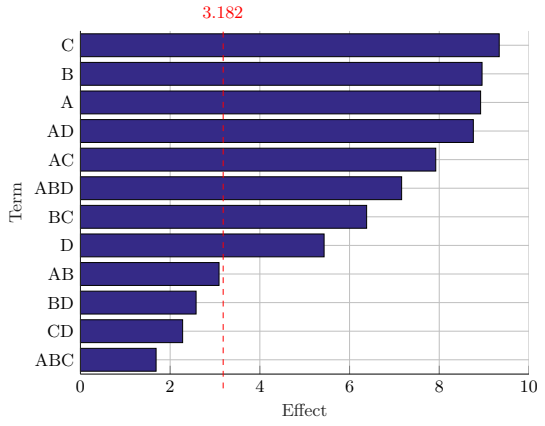

(c) Orientation accuracy

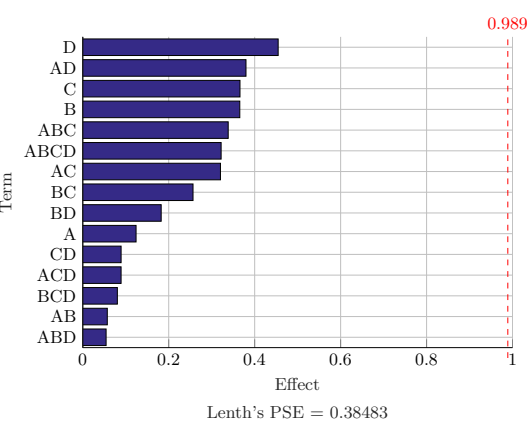

(b) Position repeatability

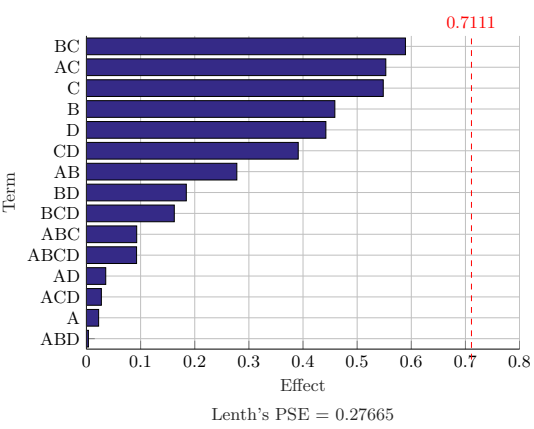

(d) Orientation repeatability

Fig. 7: Pareto effects of factors on responses: A, B, C and D stand for payload, cable tension, home MP pose and elasticity, respectively. 
Figure 8 shows the factor levels that optimize the MP accuracy and repeatability responses in term of position and orientation where the responses are equally weighted. The effect of each factor level on each response is also displayed. The composite desirability $D$ shows how well the proposed level set optimizes the four responses where individual desirability $d$ assesses how the factor set optimizes each response. The optimized choice of factor level is as followed : payload is set to "with", cable tension is set to "high", home MP pose is set to $P_{2}$ and elasticity is set to "with". The proposed set yields a good composite desirability of 0.8614 . The targeted values of the responses are $1.18 \mathrm{~mm}$ and $13.7 \mathrm{~mm}$ for position repeatability and accuracy, respectively, and $0.06 \mathrm{deg}$ and $0.62 \mathrm{deg}$ for orientation repeatability and accuracy, respectively.

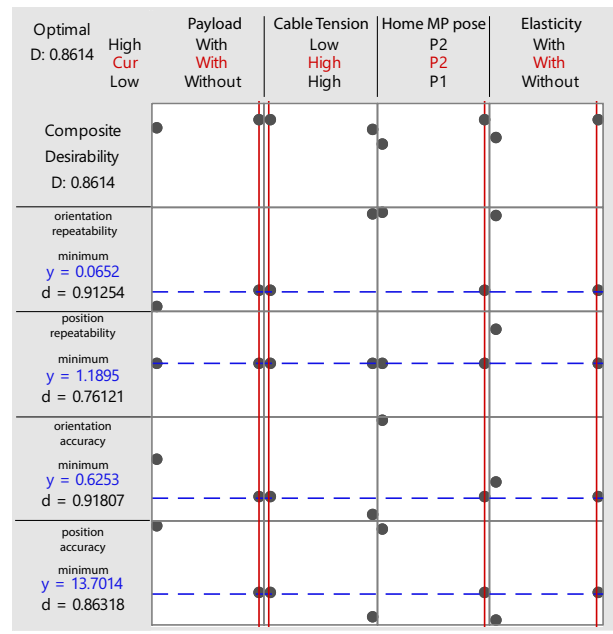

Fig. 8: Response optimization

\section{Conclusion}

This paper proposed an experimental method to evaluate the long-term running, accuracy and repeatability of a CDPR using exteroceptive measurements. In addition an investigation of the influence of factors was led using a full-factorial design of experiments. The long-term running evaluation of the prototype yields a static deviation less than $5 \mathrm{~mm}$ in terms of translation and 0.5 deg in terms of orientation while the dynamic deviation is less than $10 \mathrm{~mm}$ in terms of translation and $0.2 \mathrm{deg}$ in terms of orientation. 


\section{Acknowledgements}

This research work is part of the PACE project managed by IRT Jules Verne (French Institute in Research and Technology in Advanced Manufacturing Technologies for Composite, Metallic and Hybrid Structures). The authors wish to associate the industrial and academic partners of this project, namely, Naval Group, University of Nantes, IMT-Atlantique and CNRS. Tanguy Moro from IRT JV and the anonymous reviewers are dutifully acknowledged for their great help in using Minitab ${ }^{\circledR}$ software and improving the paper, respectively. This work is also supported by the ANR CRAFT project, grant ANR-18-CE10-0004 https://anr.fr/Project-ANR-18-CE10-0004

\section{References}

1. Aflakian, A., Safaryazdi, A., Tale Masouleh, M., Kalhor, A.: Experimental study on the kinematic control of a cable suspended parallel robot for object tracking purpose. Mechatronics 50, 160-176 (2018). https://doi.org/10.1016/j.mechatronics.2018.02.005

2. Baklouti, S., Caro, S., Courteille, E.: Elasto-dynamic model-based control of non-redundant cable-driven parallel robots. In: Arakelian, V., Wenger, P. (eds.) ROMANSY 22: robot design, dynamics and control, CISM courses and lectures, vol. 584, pp. 238-246. Springer, Cham (2019). https://doi.org/10.1007/978-3-319-78963-7_31

3. Bayani, H., Masouleh, M.T., Kalhor, A.: An experimental study on the vision-based control and identification of planar cable-driven parallel robots. Robotics and Autonomous Systems 75, 187-202 (2016). https://doi.org/10.1016/j.robot.2015.10.002

4. Dallej, T., Gouttefarde, M., Andreff, N., Michelin, M., Martinet, P.: Towards vision-based control of cable-driven parallel robots. In: 2011 IEEE/RSJ International Conference on Intelligent Robots and Systems. pp. 2855-2860. IEEE, Piscataway (Sept 2011). https://doi.org/10.1109/IROS.2011.6094591

5. Fortin-Cote, A., Cardou, P., Campeau-Lecours, A.: Improving cable driven parallel robot accuracy through angular position sensors. In: Staff, I. (ed.) 2016 IEEE RSJ International Conference on Intelligent Robots and Systems (IROS). pp. 43504355. IEEE, Piscataway (Oct 2016). https://doi.org/10.1109/IROS.2016.7759640

6. Garant, X., Campeau-Lecours, A., Cardou, P., Gosselin, C.: Improving the forward kinematics of cable-driven parallel robots through cable angle sensors. In: Gosselin, C., Cardou, P., Bruckmann, T., Pott, A. (eds.) Cable-driven parallel robots, Mechanisms and Machine Science, vol. 53, pp. 167-179. Springer Berlin Heidelberg, New York NY (2017). https://doi.org/10.1007/978-3-319-61431-1_15

7. Gosselin, C., Foucault, S.: Experimental determination of the accuracy of a three-dof cable-suspended parallel robot performing dynamic trajectories. In: Pott, A., Bruckmann, T. (eds.) Cable-Driven Parallel Robots, Mechanisms and Machine Science, vol. 32, pp. 101-112. Springer, Cham (2015). https://doi.org/10.1007/978-3-319-09489-2_8

8. Korayem, M.H., Tourajizadeh, H., Taherifar, M., Khayatzadeh, S., Maddah, M., Imanian, A., Tajik, A.: A novel method for recording the position and orientation of the end effector of a spatial cable-suspended robot and using for closed-loop 
control. The International Journal of Advanced Manufacturing Technology 72(58), 739-755 (2014). https://doi.org/10.1007/s00170-014-5681-2

9. Korayem, M.H., Yousefzadeh, M., Kian, S.: Precise end-effector pose estimation in spatial cable-driven parallel robots with elastic cables using a data fusion method. Measurement 130, 177-190 (2018). https://doi.org/10.1016/j.measurement.2018.08.009

10. Merlet, J.P.: Direct kinematics of cdpr with extra cable orientation sensors: The 2 and 3 cables case with perfect measurement and sagging cables. In: 2017 IEEE/RSJ International Conference on Intelligent Robots and Systems (IROS). pp. 6973-6978 (2017). https://doi.org/10.1109/IROS.2017.8206622

11. Merlet, J.P., Papegay, Y., Gasc, A.V.: The prince's tears, a large cable-driven parallel robot for an artistic exhibition. In: 2020 IEEE International Conference on Robotics and Automation (ICRA). pp. 10378-10383. [IEEE], [Piscataway, New Jersey] (2020). https://doi.org/10.1109/ICRA40945.2020.9197011

12. Oftadeh, R., Aref, M.M., Taghirad, H.D.: Forward kinematic analysis of a planar cable driven redundant parallel manipulator using force sensors. In: Intelligent Robots and Systems (IROS), 2010 IEEE/RSJ International Conference on. pp. 2295-2300. IEEE (2010). https://doi.org/10.1109/IROS.2010.5649471

13. Picard, E., Caro, S., Claveau, F., Plestan, F.: Pulleys and force sensors influence on payload estimation of cable-driven parallel robots. In: IROS 2018. pp. 1429-1436 (2018). https://doi.org/10.1109/IROS.2018.8594171

14. Picard, E., Caro, S., Plestan, F., Claveau, F.: Stiffness oriented tension distribution algorithm for cable-driven parallel robots. In: Lenarčič, J., Siciliano, B. (eds.) ADVANCES IN ROBOT KINEMATICS 2020, Springer Proceedings in Advanced Robotics, vol. 15, pp. 209-217. Springer, [S.l.] (2020). https://doi.org/10.1007/978-3-030-50975-0_26

15. Pott, A., Tempel, P., Verl, A., Wulle, F.: Design, implementation and longterm running experiences of the cable-driven parallel robot caro printer. In: Pott, A., Bruckmann, T. (eds.) Cable-Driven Parallel Robots, Mechanisms and Machine Science, vol. 74, pp. 379-390. Springer, Cham (2019). https://doi.org/10.1007/978-3-030-20751-9_32

16. Scalera, L., Gallina, P., Seriani, S., Gasparetto, A.: Cable-based robotic crane (cbrc): Design and implementation of overhead traveling cranes based on variable radius drums. IEEE Transactions on Robotics 34(2), 474-485 (2018). https://doi.org/10.1109/TRO.2018.2791593

17. Schmidt, V., Pott, A.: Increase of position accuracy for cable-driven parallel robots using a model for elongation of plastic fiber ropes. In: Wenger, P., Flores, P. (eds.) New trends in mechanism and machine science, Mechanisms and Machine Science, vol. 43, pp. 335-343. Springer Berlin Heidelberg, New York NY (2016). https://doi.org/10.1007/978-3-319-44156-6_34

18. Schulz, S., Seibel, A., Schlattmann, J.: Performance of an imu-based sensor concept for solving the direct kinematics problem of the stewart-gough platform. In: IROS 2018. pp. 5055-5062 (2018). https://doi.org/10.1109/IROS.2018.8594039

19. Siciliano, B., Khatib, O. (eds.): Springer Handbook of Robotics. Springer Handbooks, Springer International Publishing, Cham, 2nd edition edn. (2016)

20. Zake, Z., Chaumette, F., Pedemonte, N., Caro, S.: Robust $2 \frac{1}{2} \mathrm{~d}$ visual servoing of a cable-driven parallel robot thanks to trajectory tracking. IEEE Robotics and Automation Letters 5(2), 660-667 (2020). https://doi.org/10.1109/LRA.2020.2965033 